\title{
A vascular mass in the neck
}

\author{
Ron Eliashar, Jean-Yves Sichel, Daniel Saah, Itzhak Braverman
}

A 38-year-old man came to the ear, nose and throat clinic with a slow-growing left cervical mass. He had undergone laparotomy and excision of a functioning paraganglioma above the right adrenal gland when he was eight years old. At the age of 12 , he underwent left adrenalectomy due to a functioning paraganglioma. When he was 25 , he underwent a craniotomy because of a capillary haemangioblastoma of the cerebellum. On examination, a hard round pulsatile mass, $4 \mathrm{~cm}$ in diameter was detected in the region of the left carotid artery. Computed tomography (CT) of the neck revealed a highly vascular mass in the region of the left carotid artery bifurcation (figure). Angiography with embolization of the proximal artery supplying the mass was performed. The patient was then operated on under general anaesthesia, and the mass was removed. The postoperative period was uneventful.

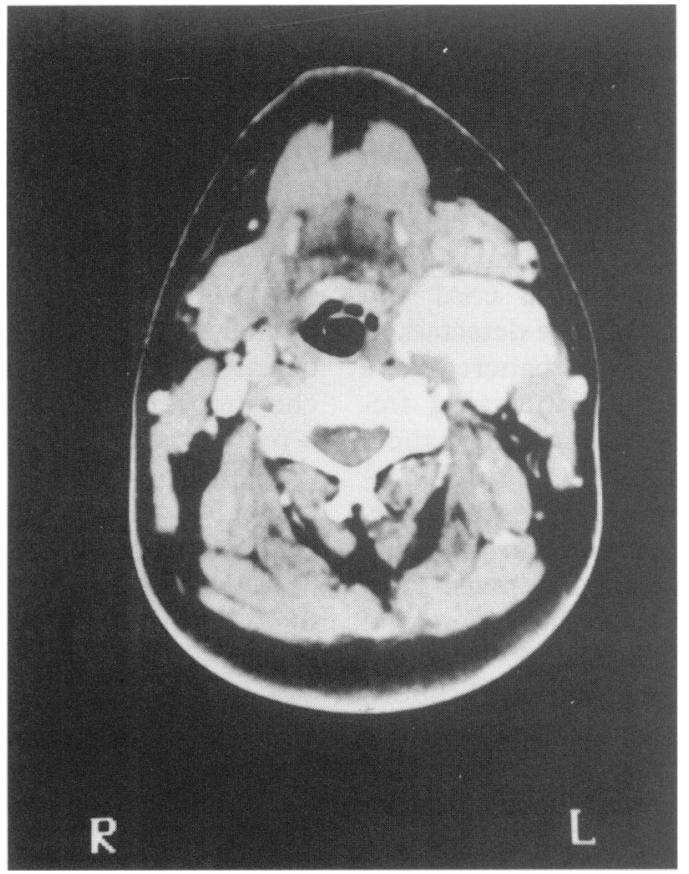

Figure Axial CT of the neck with contrast. A large enhancing lesion is noted on the left side at the level of the bifurcation of the common carotid artery

\section{Questions}

1 What is the most likely cause of the CT appearance?

2 What disease is involved and what is its mode of inheritance?

\section{Department of Otolaryngology/Head \& Neck Surgery, Hadassah University Hospital, Jerusalem, Issrael \\ R Eliashar \\ J-Y Sichel \\ I Braverman}

Accepted 4 March 1997 


\begin{abstract}
Answers
QUESTION 1

An enhancing lesion at the level of the carotid artery bifurcation is typical of a carotid body tumour. The normal carotid body is located at the bifurcation of the common carotid artery and measures $2.5 \times 5 \mathrm{~mm} .^{1}$ Carotid body tumours rarely possess vasoactive properties. ${ }^{2-4}$ Fluctuating system hypertension, palpitations and blushing are indicative of a secreting tumour. ${ }^{2}$ However, most tumours assume the form of an asymptomatic cervical mass located at the bifurcation of the common carotid artery. ${ }^{1-3}$ Large lesions may cause symptoms of pressure, eg, dysphagia, cough, hoarseness and pain. ${ }^{2,3}$ One can differentiate a carotid body tumour from a cervical lymph node by several signs ${ }^{1-3}$ : the mass, which originates from the adventitia of the carotid artery will move freely in a lateral direction and not cephalocaudally. In about $50 \%$ of patients a bruit is heard on auscultation. Carotid pulsations may also be transmitted through the mass.
\end{abstract}

\section{QUESTION 2}

The association of multiple tumours arising from the central nervous system and abdominal viscera characterises the Von HippelLindau disease which is an autosomal dominant disorder. ${ }^{5}$ Cerebellar, spinal cord and retinal haemangioblastoma may be detected, as well as cysts of the kidney, the pancreas, the liver and the epididymis. An increased frequency of hypernephroma, pancreatic cancer and phaeochromocytoma is also found. ${ }^{5}$

The family history in the present case, revealed that the mother and four brothers had suffered from phaeochromocytomas. They were all afflicted with unilateral disease and did not suffer from any other of the associated features. Two of them died from complications of the disease.

\section{Discussion}

The association of phaeochromocytoma and cerebellar haemangioblastoma has been recognised for more than 20 years ${ }^{6,7}$ and is typical of Von Hippel-Lindau disease. Cerebellar haemangioblastoma is found in about one third of the patients, ${ }^{8}$ and when it is associated with phaeochromocytoma in the same patient, its symptoms will usually appear several years after those of phaeochromocytoma. ${ }^{9}$ The term phaeochromocytoma has been used with reference to tumours of the adrenal medulla which is the largest paraganglion in the body, and to functioning tumours of the extraadrenal paraganglion system. ${ }^{4}$

Carotid body tumours are uncommon, and arise from cells in the carotid bodies which function as chemoreceptors. These cells belong to the paraganglion system which is associated with the autonomic nerve ganglia and the cranial nerves. ${ }^{4}$ Carotid body tumours are considered histologically benign, although they may induce local or distant metastasis. They usually appear in the form of a slow-
Von Hippel-Lindan disease: clinical features

- haemangioblastoma: cerebellar, spinal cord, retinal

- cysts: kidney, pancreas, liver, epididimis

- hypernephroma

- pancreatic cancer

- phaeochromocytoma

- carotid body tumour

growing cervical mass, and are almost always non-functional. ${ }^{4}$ Diagnosis is based on radiographic findings, mainly CT scan with contrast and angiography. A biopsy may cause massive bleeding and should therefore be avoided. ${ }^{3}$ Surgical excision is generally recommended except for high-risk patients or when the tumour has reached the base of the skull. For these patients radiation therapy should be considered. ${ }^{1,3}$ The Von HippelLindau disease gene is a recessive oncogene located on the short arm of chromosome 3 (3p). The clinical manifestation of the disease in members of predisposed families occurs when somatic mutations lead to the loss of chromosome $3 p$ sequences bearing the wild type allele of the Von Hippel-Lindau disease gene. ${ }^{10}$ The manifestation of the disorder is markedly variable. It ranges from patients who may suffer from one single abnormality to patients who are afflicted with multisystemic disease. Simultaneous occurrence of a phaeochromocytoma and a carotid body tumour in a patient is quite rare. To our knowledge the combination of these two abnormalities with cerebellar haemangioblastoma has not yet been described.

In the present case, the only manifestation in the other members of the afflicted family was a unilateral phaeochromocytoma. This phenomena should alert physicians to the possibility that an isolated lesion, ie, a phaeochromocytoma, a haemangioblastoma or a carotid body tumour may serve as an initial clue to the diagnosis of Von Hippel-Lindau disease. A complete evaluation of the patient and the other members of his family is then required. Screening for phaeochromocytoma should include measurements of plasma and urine catecholamines and plasma chromogranin-A, a CT-scan or magnetic resonance imaging (MRI) of the abdomen, and a meta-iodobenzylguanidine (MIBG) scintigraphy. ${ }^{11}$ Brain MRI, ophthalmoscopy and ultrasonography of the testes, should be performed for detection of any other possible lesion which may occur in Von Hippel-Lindau disease. ${ }^{11,12}$ This will enable early detection and treatment of afflicted patients. Genetic counselling is also recommended.

\section{Final diagnosis}

Carotid body tumour in a patient suffering from Von Hippel-Lindau disease.

Keywords: phaeochromocytoma, carotid-body tumour, haemangioblastoma, Von Hippel-Lindau disease 


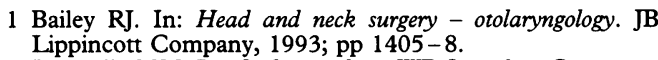

2 Paparella MM. In: Otolaryngology. WB Saunders Company, 1991; pp 2584-5.

3 Cummings CW. In: Otolaryngology - head and neck surgery. Mosby Year Book, Inc, 1993; pp 38-9, 77-8, 1595-6.

4 Mena J, Bowen JC, Hollier LH. Metachronous bilateral nonfunctional intercarotid paraganglioma (carotid body nonfunctional intercarotid paraganglioma (carotid body tumour) and functional retroperitoneal paraganglioma: report of a case and review of the literature. Surgery 1993;
114: $107-11$.

Michels VV. Investigative studies in Von-Hippel-Lindau disease. Neurofibromatosis 1988; 1: 159-63.

6 Mulholland SG, Atuk NO, Walzak MP. Familial pheochromocytoma associated with cerebellar hemangioblastoma. A case history and review of the literature. $¥ A M A 1969 ; 207$ : $1709-11$.

7 Nibbelink DW, Peters BH, McCormick WF. On the association of pheochromocytoma and cerebellar hemangioblastoma. Neurology 1969; 19: 455-60.
8 Horton WA, Wong V, Eldridge R. Von-Hippel-Lindau disease: clinical and pathological manifestations in nine families with 50 affected members. Arch Intern Med 1976; 136: $769-77$.

9 Ohnishi T, Mori S, Motozaki T, et al. Von-Hippel-Lindau disease - hemangioblastoma associated with pheochromocytoma. No Shinkei Geka 1985; 13: 757-64.

10 Tory K, Bravch $\mathrm{H}$, Linehan M, et al. Specific genetic change in tumors associated with Von-Hippel-Lindau disease. $f$ Natl Cancer Inst 1989; 81: 1097-101.

11 Neumann HP, Berger DP, Sigmund G, et al. Pheochromocytomas, multiple endocrine neoplasia type 2, and Voncytomas, multiple endocrine neoplasia type 2, and Von-

12 Furusu H, Matzuo H, Nakao K, Veda Y, Aoi W. VonHippel-Lindau disease with multiple spinal cord hemangioblastomas, syringomyelia and pheochromocytoma. Intern Med 1995; 34: 216-9.

\title{
Hyperthyroidism and dementia
}

\author{
M Dennis, S G Parker
}

An 84-year-old woman was found to be hyperthyroid in 1986 and was treated with carbimazole. Serum calcium was also raised at $2.71 \mathrm{mmol} / \mathrm{l}$ and parathyroid hormone was in the normal range. In 1992 she was admitted to an acute medical ward with a two-year history of increasing and fluctuating disorientation, forgetfulness, lethargy and constipation. Serum total calcium was found to be $3.3 \mathrm{mmol} / \mathrm{l}$, parathyroid hormone was elevated at $30 \mathrm{pmol} / 1$, but neck ultrasound was normal. As the serum calcium settled to $2.9 \mathrm{mmol} / \mathrm{l}$, the mental symptoms were felt not to be resulting from the hypercalcaemia. The patient was discharged home with additional support.

In 1994, she was referred as an out-patient to a physician for the elderly, now with a four year history of forgetfulness with a marked deterioration over the preceding seven months. She had become disorientated in time and place, suspicious, was neglecting herself, and frequently failed to recognise familiar persons. This picture was characterised by considerable day-to-day variation. She was supported in her flat by her family, five days per week home care, and mealson-wheels. Accompanying the cognitive decline were symptoms of worsening urinary incontinence, and increasing deafness. Admission was arranged for further investigation and treatment. The serum calcium fluctuated between 2.74 and $3.2 \mathrm{mmol} / \mathrm{l}$. Thyroid function, and

University of

Leicester, Leicester

General Hospital,

Leicester LE5 4PW, UK

Division of Psychiatry

for the Elderly

M Dennis

Division of Medicine

for the Elderly

SG Parker

Accepted 20 March 1997 investigations to exclude malignancy and delirium were all normal. At this time she appeared alert, though disorientated in time, with poor recall and concentration.

\section{Questions}

1 What is the most probable diagnosis?

2 How can a causal link between the diagnosis and the presence of cognitive impairment be established?

3 What is the management? 